\title{
Ranking Self-reported Gastrointestinal Side Effects of Pharmacotherapy in Sarcoidosis
}

\author{
M. Drent ${ }^{1,2,3}$ - V. L. J. Proesmans $3^{3,4} \cdot$ M. D. P. Elfferich ${ }^{3} \cdot$ N. T. Jessurun ${ }^{3,5} \cdot$ S. M. G. de Jong ${ }^{3,4} \cdot$ N. M. Ebner ${ }^{3,4}$. \\ E. D. O. Lewis ${ }^{3,4} \cdot$ A. Bast ${ }^{2,3,4}$
}

Received: 12 September 2019 / Accepted: 6 January 2020 / Published online: 20 January 2020

(c) The Author(s) 2020

\begin{abstract}
Background Clinical manifestations of sarcoidosis vary widely, depending on the intensity of the inflammation and the organ systems affected. So far, no curative treatment exists; the disease can only be suppressed. All treatment options cause side effects affecting quality of life. The aim of this study was to establish and rank the prevalence of self-reported gastrointestinal side effects of drugs used in the treatment of sarcoidosis.

Methods A cross-sectional web-based anonymous survey about complaints and side effects was conducted among sarcoidosis patients in the Netherlands, United Kingdom, and United States of America.

Results Of the participants, $70 \%$ were being treated with one or more drugs. The most important reported side effect was weight gain, associated with increased appetite among prednisone users (as monotherapy as well as in combination with other drugs). Methotrexate (MTX) users especially experienced nausea, with monotherapy as well as combination therapy. Vomiting and weight loss were most prominent among azathioprine and mycophenolate mofetil (MMF) users, whereas diarrhoea was frequently mentioned by MMF and MTX users. The reported side effects of hydroxychloroquine were generally rather mild.

Conclusion The current study ranked the gastrointestinal side effects associated with pharmacotherapy in sarcoidosis patients. Pharmacotherapy does have multiple gastrointestinal side effects. The strongest association between a reported side effect and drug use was that of weight gain associated with increased appetite among prednisone users. It would therefore be useful for future research to look further into dietary interventions to counter these side effects and reduce their burden.
\end{abstract}

Keywords Gastrointestinal side effects · Glucocorticoids · Methotrexate · Pharmacotherapy · Sarcoidosis · Side effects · Treatment

\section{Introduction}

M. Drent and V. L. J. Proesmans contributed equally to this work.

M. Drent

m.drent@antoniusziekenhuis.nl

1 Department of Pharmacology and Toxicology, FHML, Maastricht University, Maastricht, The Netherlands

2 Department of Pulmonology, ILD Center of Excellence, St. Antonius Hospital, Koekoekslaan 1, 3435 CM Nieuwegein, The Netherlands

3 ild care foundation Research Team, Ede, The Netherlands

4 Venlo Campus, Maastricht University, Venlo, The Netherlands

5 Netherlands' Pharmacovigilance Centre Lareb, 's Hertogenbosch, The Netherlands
Sarcoidosis is a multisystem inflammatory disorder of unknownetiology. The disease is characterized by the formation of non-caseating granulomas in various organ systems, mainly the lungs and lymphatic system, but any organ can be involved [1]. The exact cause of sarcoidosis is still unknown, but is likely to depend on both genetic and environmental factors, probably antigen-driven. The natural history and prognosis of sarcoidosis are highly variable. Within 3 years of diagnosis, over $50 \%$ of patients have achieved remission, while within a decade approximately one-third of patients have persistent disease, leading to a significant burden on their lives $[1,2]$. The management of sarcoidosis is challenging due to the heterogeneity of its organ manifestations and its clinical course, as well as the potential side effects of 
current immunosuppressive therapy. The lack of approved drugs tested in randomized controlled trials hampers the development of standardized treatment protocols for sarcoidosis [1]. As a general rule, deterioration that threatens any organ function warrants treatment. Systemic sarcoidosis treatment should generally be given to prevent end-organ damage.

There are three lines of therapy for sarcoidosis. Oral glucocorticoids (GCs) are the initial first-line therapy for symptomatic patients. Non-specific immunosuppression with prednisone remains the first-choice systemic therapeutic option [1]. Second-line agents in sarcoidosis include MTX, azathioprine (AZA), leflunomide, and hydroxychloroquine (HCQ), although the available evidence supporting their use, like that of prednisone, is limited. Of the secondline agents for sarcoidosis, MTX has been the most widely studied and guidelines have been established [3]. MTX has been demonstrated to be effective and less toxic than AZA $[3,4]$. Leflunomide is similar to MTX in action but with a different toxicity profile [5-7]. It is associated with less nausea and pulmonary toxicity [8], but it can cause peripheral neuropathy. More recently, mycophenolate mofetil (MMF) was reported to be useful when other drugs had to be discontinued due to toxicity; however, evidence for the effectiveness of this product is rather limited [9]. It was suggested to be successful in neurosarcoidosis and cutaneous sarcoidosis [10]. HCQ and chloroquine are antimalarial agents that have proved useful to treat skin manifestations [11] and abnormalities of calcium metabolism [12]. Third-line treatment consists of biologicals (tumor necrosis factor-alpha (TNF$\alpha$ ) inhibitors), and is currently reserved for patients nonresponsive to first- or second-line treatment. Guidelines have been established to help identify which patients to treat, and include dosing and monitoring schemes [13]. Infliximab is the most widely studied and administered monoclonal antibody for the treatment of various manifestations of severe sarcoidosis [13]. Adalimumab has also been used, although to a lesser extent $[13,14]$. Nowadays, biosimilars are commonly used and have proved to be effective and safe as well [15-17].

The drugs currently used to treat various manifestations of sarcoidosis tend to cause side effects, including gastrointestinal side effects, which then further increase the burden of this disease [1]. Since the importance of patient participation in healthcare decisions has been increasingly acknowledged [18], and studies from a patient perspective are important [19], we conducted an online survey of selfreported gastrointestinal side effects.

The aim of this study was to establish and rank the prevalence of self-reported gastrointestinal side effects of drugs used in the treatment of sarcoidosis.

\section{Methods}

\section{Study Design}

In cooperation with the Dutch Sarcoidosis Patient Society (Sarcoidose.nl), the ild care foundation designed a questionnaire about side effects of drugs used to treat sarcoidosis-related problems. The questionnaire was used in a cross-sectional web-based anonymous survey conducted from June 2018 to December 2018 among a sample of sarcoidosis patients in the Netherlands, United Kingdom, and United States. This study was performed in accordance with the Declaration of Helsinki and its amendments.

\section{Study Subjects and Procedure}

Patients were recruited through membership of the Dutch Sarcoidosis Society, Sarcoidose.nl, via the society's newsletter and through an advertisement at the ILD Center of Excellence at Nieuwegein in the Netherlands, as well as through membership of SILA (Sarcoidosis and Interstitial Lung Association) in the UK and FSR (Stop Sarcoidosis Research Foundation) in the USA. Participating patients were proficient in Dutch or English and had internet access. Patients were enrolled in NL, UK, and USA without incentives, since the survey was anonymous. The survey was developed using the online questionnaire tool Surveymonkey (www.surveymonkey.com). The questions concerned demographics (gender, age, duration of sarcoidosis), the burden of sarcoidosis and symptoms experienced by the patients, the use of medication, and side effects. Respondents were asked to complete the questionnaire even if they had never experienced any problems with their drugs. Those patients who reported not using any drugs to treat sarcoidosis were regarded as controls.

\section{Statistical Analysis}

All statistical analyses were performed using SPSS statistical software (22.0 for Windows; SPSS Inc., IL, USA). The adverse side effects of GCs, MTX, TNF- $\alpha$ inhibitors, AZA, HCQ, and mycophenolate mofetil (MMF) were tested by assessing the variables nausea, vomiting, diarrhoea, weight loss, weight gain, appetite loss, loss of taste perception, loss of smell perception, and dry mouth, using a logistic regression analysis with a logit link. In this logistic regression, the side effects were used as response variables, while the various drugs were used as explanatory variables. The data were analyzed and summarized 
in a forest plot developed using Metaexcel, an add-on for Excel.

\section{Results}

Table 1 summarizes the demographic and clinical data of our sarcoidosis sample. In total, 937 patients completed the questionnaire: 282 patients were using no drugs, 416 used prednisone either alone or in combination, 272 were using MTX, 128 TNF- $\alpha$ inhibitors, 53 AZA, 107 HCQ, and 29 MMF.

Table 2 presents the numbers of participants using a single drug or a combination of two drugs. Sixty patients in our sarcoidosis sample were being treated with more than two drugs, and were not included in Table 2, while $282(30 \%)$ of our sample were currently not being treated for their sarcoidosis with any of the above-mentioned drugs. Of the drug
Table 1 Summary of the demographic and clinical data of the three sarcoidosis samples

\begin{tabular}{|c|c|c|c|c|}
\hline & The Netherlands & United Kingdom & United States & Total \\
\hline Number & 646 & 37 & 254 & 937 \\
\hline Age, mean $\pm S D$ & $54 \pm 11$ & $53 \pm 10$ & $55 \pm 10$ & $55 \pm 11$ \\
\hline Male (\%) & 38.2 & $18.9 *$ & 30.0 & 35.3 \\
\hline \multicolumn{5}{|l|}{ Time since diagnosis (\%) } \\
\hline$<1$ year & 11.8 & 13.5 & $4.0^{*}$ & 9.8 \\
\hline $1-2$ year & 12.9 & 21.6 & 10.8 & 12.7 \\
\hline $2-5$ year & $23.8 *$ & 35.1 & 32.7 & 26.6 \\
\hline$>5$ year & 51.5 & $29.7 *$ & 52.6 & 50.9 \\
\hline BMI $\left(\mathrm{kg} / \mathrm{m}^{2}\right)$, mean \pm SD & $28.7 \pm 5.8$ & $28.7 \pm 6.1$ & $31.5 \pm 7.9^{*}$ & $29.4 \pm 6.5$ \\
\hline Smoker (\%) & 4.5 & 11.1 & 5.3 & 5.0 \\
\hline Non-smoker (\%) & 36.8 & $47.2 *$ & 29.9 & 35.4 \\
\hline Former smoker (\%) & 58.7 & 41.7 & 64.8 & 59.6 \\
\hline Non-drug users (\%) & 33.1 & 32.4 & $22.0 *$ & 30 \\
\hline \multicolumn{5}{|l|}{ Drug users (\%) } \\
\hline Prednisone & $40.4 *$ & 59.5 & 52.4 & 44.4 \\
\hline Methotrexate & 28.9 & 16.2 & 31.1 & 29.0 \\
\hline TNF- $\alpha$ inhibitors & 13.2 & 2.7 & 16.5 & 13.7 \\
\hline Azathioprine & 4.3 & 5.4 & $9.1^{*}$ & 5.7 \\
\hline Hydroxychloroquine & 9.1 & 10.8 & $17.3 *$ & 11.4 \\
\hline Mycophenolate mofetil & 2.0 & 0 & $6.3^{*}$ & 3.1 \\
\hline \multicolumn{5}{|c|}{ Duration of use of current drug (\%) } \\
\hline$<6$ months & 16.2 & 16.0 & 12.5 & 15.2 \\
\hline $6-12$ months & 11.7 & 12.0 & 11.1 & 11.6 \\
\hline$>1$ year & 72.0 & 72.0 & 76.4 & 73.3 \\
\hline
\end{tabular}

Data are expressed as mean $\pm \mathrm{SD}$ or percentage if appropriate

$B M I$ body mass index

*Value differs significantly from the two other countries $(p<0.05)$
Table 2 Number of sarcoidosis patients studied using a single drug or a combination of two drugs, subdivided into glucocorticoids (GCs) like prednisone, methotrexate (MTX), TNF-alpha inhibitors (TNF- $\alpha-i)$, azathioprine (AZA), hydroxychloroquine (HCQ), and mycophenolate mofetil (MMF)

\begin{tabular}{lrrrrrr}
\hline & GC & MTX & TNF- $\alpha-i$ & AZA & HCQ & MMF \\
\hline GC & 206 & 88 & 21 & 18 & 18 & 10 \\
MTX & 88 & 89 & 38 & 26 & 0 & 0 \\
TNF- $\alpha-i$ & 21 & 38 & 3 & 13 & 2 & 3 \\
AZA & 18 & 0 & 2 & 2 & 0 & 39 \\
HCQ & 18 & 13 & 3 & 0 & 0 \\
MMF & 10 & 0 & & & 7 \\
\hline
\end{tabular}


users ( $n=655,70 \%), 380$ (58\%) were using one drug and $214(33 \%)$ were using two drugs (see Table 2). Forty-nine (7\%) patients were being treated with combinations of three drugs and 11 (2\%) with four or five drugs. The combination of prednisone with MTX was most common (88 patients out of those using two drugs, 32 out of those using three drugs [15 combined with TNF- $\alpha$-inhibitors, 3 combined with AZA and 14 with HCQ], and 9 patients out of those using four drugs).

Two forest plots summarize the occurrence of gastrointestinal side effects, ranked for the different drugs used for the treatment of sarcoidosis (Fig. 1a, b). Prednisone use was associated with increased appetite (OR 9.26) and with weight gain (OR 5.68). This effect was also evident for those using combinations with the other drugs (data not shown).

MTX users reported suffering most from nausea (OR 4.35). This effect occurred both with monotherapy and with combinations with other drugs (data not shown). All other drugs except TNF- $\alpha$-inhibitors also had nausea as a side-effect, although to a lesser extent. AZA and MMF were especially associated with vomiting (OR 3.04 and 4.50, respectively). MMF use contributed most to diarrhoea complaints (OR 3.11). AZA, HCQ, and MMF users showed significantly more weight loss, with odds ratios ranging from 2.39 to 3.95

Weight gain was associated with increased appetite $(r=0.489, p<0.001)$, while weight loss was associated with loss of appetite $(r=0.468)$, as well as nausea $(r=0.193)$, vomiting $(r=0.184)$, and diarrhoea $(r=0.133$; all $p$ 's $<0.001)$.

The spider plots (Fig. 2a, b) show significantly more appetite loss, nausea, and diarrhoea complaints among the treatment groups compared to the controls $(p<0.05)$. Vomiting also occurred significantly more among the different treatment groups, except for prednisone monotherapy. The same goes for the weight gain complaints, which increased in all treatment groups except the TNF- $\alpha$-inhibitor monotherapy $(p<0.05)$.

Comparing the different drug groups, Fig. 2a shows that prednisone monotherapy as well as prednisone combined with MTX increased appetite and weight gain complaints compared to MTX monotherapy $(p<0.05)$. As regards nausea, MTX monotherapy and combination therapy with prednisone caused a significantly higher burden than prednisone monotherapy.

Among the different TNF- $\alpha$ inhibitor treatment options (Fig. 2b), the combination of prednisone and TNF- $\alpha$ inhibitors significantly increased appetite and weight gain complaints $(p<0.05)$. Diarrhoea complaints were also higher in the TNF- $\alpha$ inhibitor group, but the difference was not significant $(p=0.05)$.

Duration of illness, age, and gender mainly had effects on the prescription of drugs. No important relationships were found between these parameters and the side effects. A more detailed evaluation of the effect of prednisone on weight and appetite showed that these effects were smaller in men than in women, and that age was negatively correlated with the magnitude of the effect, but that the duration of the disease did not have an influence. As regards the observed effect of MTX on nausea, gender did not play a role in this, nor did the disease duration affect the nausea effect elicited by MTX. Age was negatively correlated with the nausea effect of MTX, as younger patients experienced more nausea as a side effect.

\section{Discussion}

This study has assessed the associations between drugs and side effects of pharmacotherapy among 932 sarcoidosis patients, and ranked their self-reported gastrointestinal side effects. The most frequently reported side effect was weight gain, associated with increased appetite, among prednisone users. Nausea was experienced by MTX users (as monotherapy as well as in combination with other drugs). Both vomiting and weight loss were most prominent in AZA and MMF users, whereas diarrhoea was mainly reported by MMF and MTX users. The reported side effects of HCQ were generally rather mild.

Sarcoidosis is characterized by inflammation, and current treatment mainly consists of anti-inflammatory drugs. Since the cause of the disease is still unknown, it is important to carefully evaluate the treatment of choice, considering its possible benefits and drawbacks. In line with previous studies, the most important side effect related to prednisone use in this study was weight gain and increased appetite [20-22]. These effects were smaller in men than in women. This is probably due to a difference in BMI. This is problematic because obesity is an increasing health problem, also for patients with sarcoidosis [23, 24]. Apart from prednisone, all other drugs ranked in this study did not result in substantial weight gain. Moreover, prolonged prednisone therapy is associated with other significant side effects as well, such as diabetes, infections, fluid retention, muscle weakness, glaucoma, cataracts, insomnia, mood swings, personality changes, osteoporosis, and osteonecrosis of the femoral head [21]. The risk of side effects appears to depend on both the cumulative dose and the duration of prednisone use [21], whereas the efficacy of prednisone was not found to be related to the dosage [25]. Given the severe adverse events accompanying long-term treatment with prednisone, timely use (before an unacceptable increase in BMI) of an appropriate cytotoxic agent with steroid-sparing potency is to be recommended. In case of an increased BMI it might even be useful to start straight away with a GC-sparing agent. Another approach might be to consume appropriate 
Fig. 1 a Forest plot of gastrointestinal side effects (nausea, vomiting, and diarrhoea), ranked for the various drugs used in the treatment of sarcoidosis, in comparison to non-drug users. b Forest plot of gastrointestinal side effects (weight loss, appetite loss, appetite increase, and weight gain) ranked for the different drugs used in the treatment of sarcoidosis, in comparison to non-drug users

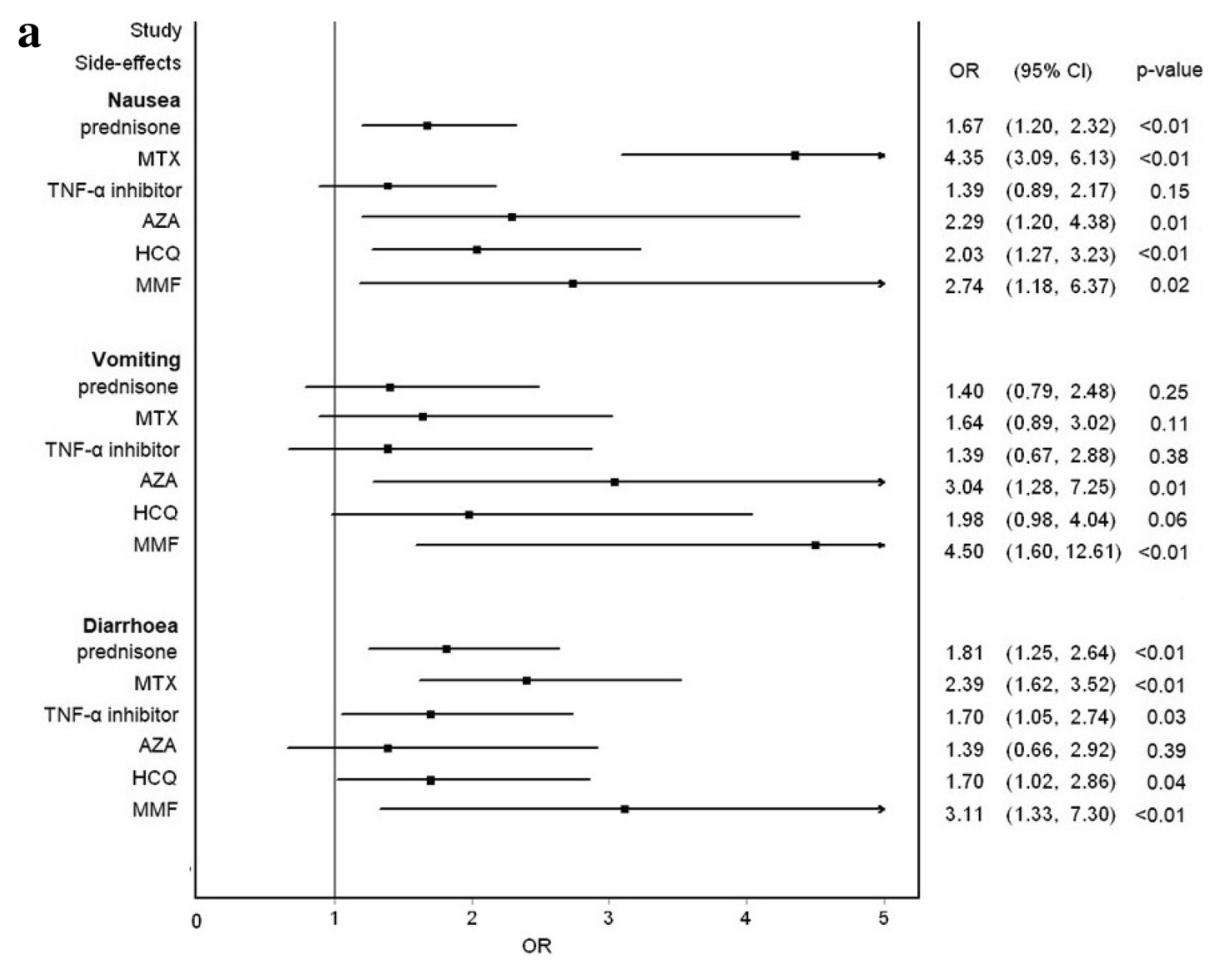

b

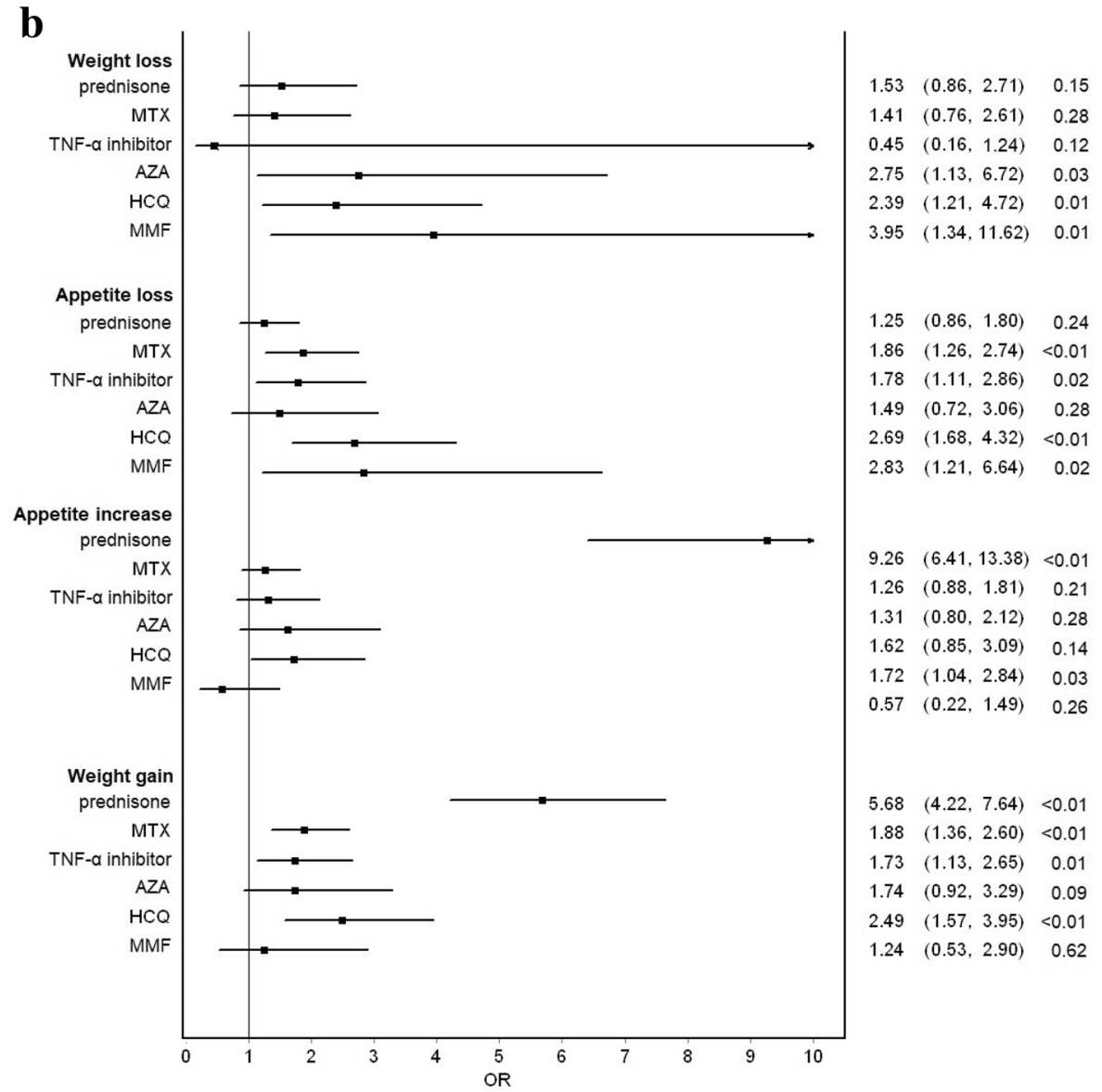




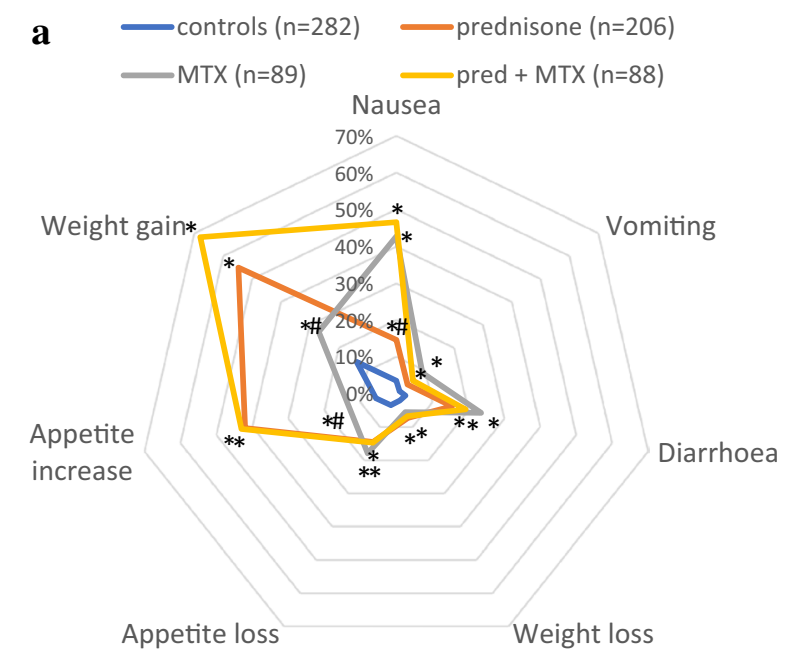

b

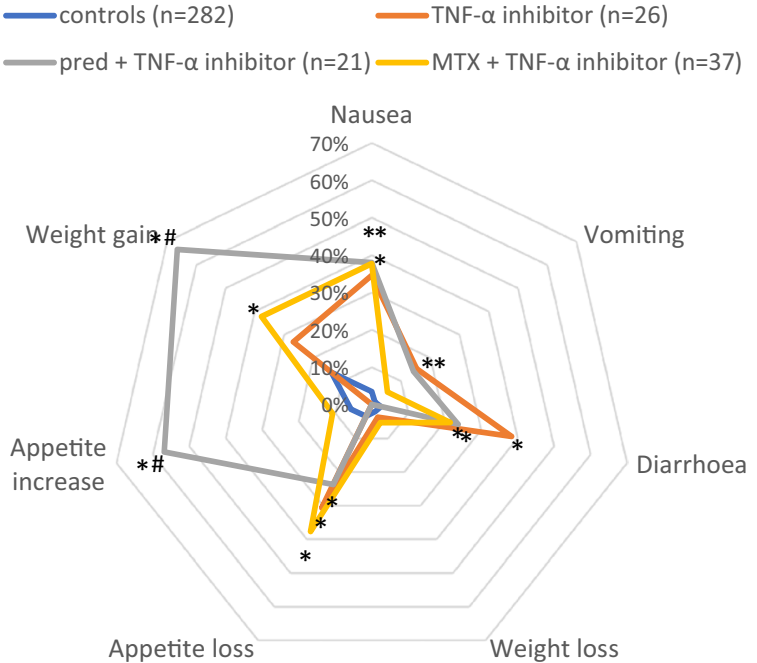

Fig. 2 a Spider plot of gastrointestinal side effects of prednisone, methotrexate (MTX), and the combination of prednisone and MTX therapy in sarcoidosis. *Group differs significantly $(p<0.05)$ from the control group (non-drug users). ${ }^{\#}$ Group differs significantly from the other drug groups $(p<0.05)$. b. Spider plot of gastrointestinal side effects of TNF- $\alpha$ inhibitors: monotherapy or in combination with prednisone or methotrexate (MTX). *Group differs significantly $(p<0.05)$ from the control group (non-drug users). "Group differs significantly from the other drug groups $(p<0.05)$

flavonoid-rich food in conjunction with corticosteroids, which has appeared to increase the efficacy of corticosteroids, thereby reducing the dose required for anti-inflammatory action, with less risk of developing side effects [26].

The existence of oxidative stress in sarcoidosis patients is evident from the decrease in overall plasma antioxidant values. Clearly, antioxidant supplementation, such as quercetine, might be helpful to restore the antioxidant levels in these patients [27-29]. It has even been suggested that the anti-inflammatory effect of flavonoids might result from their maintaining effect on the ability of cortisol to cope with pro-inflammatory triggers and the prevention of overreactive inflammatory processes [26].

Generally, MTX and AZA are regarded as the secondline treatments, with MTX being preferred to AZA because of lower rates of discontinuation [14]. In terms of gastrointestinal side effects, MTX was associated with increased nausea, diarrhoea, decreased appetite, weight gain, and dry mouth $(p<0.05)$. The significantly increased nausea but not vomiting is in line with previous data, as is the occurrence of diarrhoea [4]. Weight gain complaints were also found in studies of rheumatoid arthritis (RA) patients who used MTX [30]. The OR for weight gain was 1.88 among MTX users, which is significantly lower than the OR for weight gain among prednisone users (5.68). Hence, MTX could reduce weight gain when used in conjunction with prednisone as a corticosteroid sparing drug, as reported in a previous study [31]. AZA was associated with nausea and vomiting, which is in line with previous research [32].

TNF- $\alpha$ inhibitors are regarded as third-line treatment. The current study showed that TNF- $\alpha$ inhibitor use was associated with decreased appetite, weight gain, and diarrhoea (with or without concurrent drug use). However, the largest study using infliximab in sarcoidosis, by Baughman et al., reported no gastrointestinal side effects [33]. Meanwhile, gastrointestinal adverse drug reactions associated with the use of infliximab have been recognized and even included in the summary of product characteristics of Remicade ${ }^{\circledR}$ [34]. Sfriso et al. reported weight gain in patients with RA under treatment with TNF- $\alpha$ inhibitors [35]. A previous study among patients at a rheumatology clinic also reported weight gain in patients with various indications treated with TNF- $\alpha$ inhibitors [36]. However, no significant changes in appetite were noted among this group [36].

HCQ use was associated with nausea, diarrhoea, decreased appetite, and weight loss in some cases, but increased appetite and weight gain in others. Weight gain among HCQ users has been suggested to be associated with a reduction of gut microbiota caused by the drug [37]. Mycophenolate mofetil was associated with nausea, vomiting, and diarrhoea, which was also found in previous studies $[9,10]$. Weight loss, decreased appetite, and loss of taste perception were also noted among the patients in the present study.

Although drug-induced taste disturbances are common, they have failed to attract the attention of most clinicians. Similarly, taste disorders have been largely ignored by pharmaceutical companies during preclinical drug development [38]. Only the summary of product characteristics of MTX tablets mentions taste disorders as a possible adverse drug reaction. Recently, Schiffman published a review about the influence of medications on taste and smell [39]. However, the incidence and prevalence of medication-induced 
chemosensory disorders referred to in this review related to the use of immunosuppressant drugs, and to other drugs used to treat sarcoidosis are lacking. In the present study the influence of all reported drugs on taste and smell perception appeared to be rather mild and minor compared to the other gastrointestinal side effects. This was in accordance with previous studies, which reported a possible effect of corticosteroids on taste perception [40]. Dry mouth was most prominent in prednisone users, whereas loss of taste perception was most prominent in MMF users.

The large patient group included in our study enabled us to attribute a clear statistical significance ( $p$-value) to the actual contribution of the side effects induced by drug treatment, as indicated in the Forest plot of Fig. 1. Moreover, the results are consistent with those of other studies which suggest that many of the problems encountered are related to the medications rather than the sarcoidosis.

\section{Limitations}

One of the limitations of this study is that information about disease severity was lacking, so the impact of disease severity on the reported side effects could not be established. Another limitation is that the symptoms were self-reported and not verified by a health care professional. However, this is what patients experience and associate with the drugs used for their sarcoidosis.

\section{Recommendations}

The data from our study show that all drugs reported on cause some burden of gastrointestinal side effects. Therefore, it would be useful to look into possible dietary interventions to minimize this burden, as well as the use of other drugs to counter the side effects. Overweight and obese patients should avoid GC and should be considered for second-line treatment directly. Strategies to manage gastrointestinal side effects caused by one of the sarcoidosis drugs start with the advice to take the tablets during a meal, not on an empty stomach, which might reduce the peak dose. In the case of MTX-induced gastrointestinal side effects, including mucositis, adding folic acid to the diet as well as splitting the oral dose should be considered, provided the total MTX dose is ingested within a 12-h period. Parenteral administration or an alternative immunosuppressive drug should be considered in case of persistent intolerance [3]. In case of incessant diarrhoea, the next step may be rehydration and anti-diarrhoeal medication, for example loperamide, and in case of constant nausea, anti-emetics. Furthermore, antacids are recommended for indigestion [41]. Although there is no 'one-size-fits-all' policy for persistent side effects, supervised dose reduction and re-titration may be required once the symptoms have subsided.

Our findings can be used to support patients and their care providers in the choice of drugs with regard to gastrointestinal side effects. With previous research findings already pointing at the possible role of food components and additives in inflammatory lung diseases [27-29], it could be interesting to explore the possibilities of implementing dietary interventions in sarcoidosis treatment in order to reduce the side effects or counter the disease.

\section{Conclusion}

The current study ranked the gastrointestinal side effects associated with pharmacotherapy in sarcoidosis patients, in terms of frequency. The currently used pharmacotherapy causes multiple gastrointestinal side effects. The most important reported side effect was weight gain associated with increased appetite among prednisone users (both as monotherapy and in combination with other drugs). Since obesity is still an increasing health problem, alternatives that can be used as first-line treatment in sarcoidosis are urgently needed. The next most important side effect appeared to be nausea, experienced especially by MTX users (as monotherapy as well as in combination with other drugs). It would therefore be useful for future research to look further into dietary interventions to counter these side effects and reduce their burden.

Acknowledgements The authors would like to thank the Dutch Sarcoidosis Society: Sarcolidose.nl (www.sarcoidose.nl), the Foundation for Sarcoidosis Research (FSR: www.stopsarcoidosis.org), the patient society of the US and the UK patient society SarcoidosisUK (www. sarcoidosisuk.org), as well as all participants of this study for all their efforts to make this project possible.

Author Contributions All authors were involved in the study design and data collection. MD and VP contributed equally. VP, MD, and $\mathrm{AB}$ analyzed the data and drafted the manuscript. $\mathrm{ME}, \mathrm{SJ}, \mathrm{NE}$, and $\mathrm{EL}$ conducted the survey. ME, and $\mathrm{NJ}$ revised the manuscript critically for important intellectual content. All authors read and approved the final manuscript.

Funding This study was supported by a research grant from the ild care foundation: www.ildcare.nl. The study sponsor had no involvement in the study design; in the collection, analysis, and interpretation of data; in the writing of the manuscript; or in the decision to submit the manuscript for publication.

Data Availability The datasets used and/or analyzed during the current study are available from the corresponding author on reasonable request. 


\section{Compliance with Ethical Standards}

Conflict of interest The authors declare that they have no competing interests.

Ethical Approval In accordance with the Dutch Act on Human Subjects Medical Research, the Medical Ethics Committee of St. Antonius Hospital Nieuwegein waived formal approval.

Open Access This article is licensed under a Creative Commons Attribution 4.0 International License, which permits use, sharing, adaptation, distribution and reproduction in any medium or format, as long as you give appropriate credit to the original author(s) and the source, provide a link to the Creative Commons licence, and indicate if changes were made. The images or other third party material in this article are included in the article's Creative Commons licence, unless indicated otherwise in a credit line to the material. If material is not included in the article's Creative Commons licence and your intended use is not permitted by statutory regulation or exceeds the permitted use, you will need to obtain permission directly from the copyright holder. To view a copy of this licence, visit http://creativecommons.org/licenses/by/4.0/.

\section{References}

1. Grunewald J, Grutters JC, Arkema EV, Saketkoo LA, Moller DR, Muller-Quernheim J (2019) Sarcoidosis. Nat Rev Dis Primers $5(1): 45$

2. Drent M, Strookappe B, Hoitsma E, De Vries J (2015) Consequences of sarcoidosis. Clin Chest Med 36(4):727-737

3. Cremers JP, Drent M, Bast A, Shigemitsu H, Baughman RP, Valeyre D, Sweiss NJ, Jansen TL (2013) Multinational evidencebased world association of sarcoidosis and other granulomatous disorders recommendations for the use of methotrexate in sarcoidosis: integrating systematic literature research and expert opinion of sarcoidologists worldwide. Curr Opin Pulm Med 19(5):545-561

4. Vorselaars ADM, Wuyts WA, Vorselaars VMM et al (2013) Methotrexate vs azathioprine in second-line therapy of sarcoidosis. Chest 144(3):805-812

5. Korsten P, Strohmayer K, Baughman RP, Sweiss NJ (2016) Refractory pulmonary sarcoidosis-proposal of a definition and recommendations for the diagnostic and therapeutic approach. Clin Pulm Med 23(2):67-75

6. Baughman RP, Grutters JC (2015) New treatment strategies for pulmonary sarcoidosis: antimetabolites, biological drugs, and other treatment approaches. Lancet Respir Med 3(10):813-822

7. Vorselaars AD, van Moorsel CH, Deneer VH, Grutters JC (2013) Current therapy in sarcoidosis, the role of existing drugs and future medicine. Inflamm Allergy Drug Targets 12(6):369-377

8. Cannon GW, Holden WL, Juhaeri J, Dai W, Scarazzini L, Stang P (2004) Adverse events with disease modifying antirheumatic drugs (DMARD): a cohort study of leflunomide compared with other DMARD. J Rheumatol 31(10):1906-1911

9. Hamzeh N, Voelker A, Forssen A, Gottschall EB, Rose C, Mroz P, Maier LA (2014) Efficacy of mycophenolate mofetil in sarcoidosis. Respir Med 108(11):1663-1669

10. Bitoun S, Bouvry D, Borie R et al (2016) Treatment of neurosarcoidosis: a comparative study of methotrexate and mycophenolate mofetil. Neurology 87(24):2517-2521

11. Baughman RP, Lower EE (2007) Evidence-based therapy for cutaneous sarcoidosis. Clin Dermatol 25(3):334-340
12. Baughman RP, Janovcik J, Ray M, Sweiss N, Lower EE (2013) Calcium and vitamin D metabolism in sarcoidosis. Sarcoidosis Vasc Diffuse Lung Dis 30(2):113-120

13. Drent M, Cremers JP, Jansen TL, Baughman RP (2014) Practical eminence and experience-based recommendations for use of TNFalpha inhibitors in sarcoidosis. Sarcoidosis Vasc Diffuse Lung Dis 31(2):91-107

14. James WE, Baughman R (2018) Treatment of sarcoidosis: grading the evidence. Expert Rev Clin Pharmacol 11(7):677-687

15. Schimmelpennink MC, Vorselaars ADM, van Beek FT, Crommelin HA, Deneer VHM, Keijsers RGM, Veltkamp M (2018) Efficacy and safety of infliximab biosimilar Inflectra ${ }^{\circledR}$ in severe sarcoidosis. Respir Med 138:S7-S13

16. Merinopoulos D, Hayes F, Gallagher DA, Dasgupta B (2017) A case report of neurosarcoidosis successfully treated with an infliximab biosimilar after a relapse while on dual therapy. Clin Exp Rheumatol 35(2):356-357

17. Veltkamp M, Drent M, Baughman RP (2016) Infliximab or biosimilars in sarcoidosis; to switch or not to switch? Sarcoidosis Vasc Diffuse Lung Dis 32(4):280-283

18. Moor CC, Gur-Demirel Y, Wijsenbeek MS (2019) Feasibility of a comprehensive home monitoring program for sarcoidosis. J Pers Med 9(2):23

19. van Helmondt SJ, Polish LB, Judson MA, Grutters JC (2019) Patient perspectives in sarcoidosis. Curr Opin Pulm Med 25(5):478-483

20. van Everdingen AA, Jacobs JW, Siewertsz Van Reesema DR, Bijlsma JW (2002) Low-dose prednisone therapy for patients with early active rheumatoid arthritis: clinical efficacy, disease-modifying properties, and side effects: a randomized, double-blind, placebo-controlled clinical trial. Ann Intern Med 136(1):1-12

21. Khan NA, Donatelli CV, Tonelli AR, Wiesen J, Ribeiro Neto ML, Sahoo D, Culver DA (2017) Toxicity risk from glucocorticoids in sarcoidosis patients. Respir Med 132:9-14

22. Berthon BS, MacDonald-Wicks LK, Wood LG (2014) A systematic review of the effect of oral glucocorticoids on energy intake, appetite, and body weight in humans. Nutr Res 34(3):179-190

23. Cremers JP, Drent M, Elfferich MD, Nelemans PJ, Wijnen PA, Witteman BJ, Schols AM (2013) Body composition profiling in a Dutch sarcoidosis population. Sarcoidosis Vasc Diffuse Lung Dis 30(4):289-299

24. Cozier YC, Govender P, Berman JS (2018) Obesity and sarcoidosis: consequence or contributor? Curr Opin Pulm Med 24(5):487-494

25. Broos CE, Poell LHC, Looman CWN et al (2018) No evidence found for an association between prednisone dose and FVC change in newly-treated pulmonary sarcoidosis. Respir Med 138:S31-S37

26. Ruijters EJ, Haenen GR, Weseler AR, Bast A (2014) The cocoa flavanol (-)-epicatechin protects the cortisol response. Pharmacol Res 79:28-33

27. Bast A, Semen KO, Drent M (2018) Nutrition and corticosteroids in the treatment of sarcoidosis. Curr Opin Pulm Med 24(5):479-486

28. Boots AW, Drent M, de Boer VC, Bast A, Haenen GR (2011) Quercetin reduces markers of oxidative stress and inflammation in sarcoidosis. Clin Nutr 30(4):506-512

29. de Boer A, van de Worp W, Hageman GJ, Bast A (2017) The effect of dietary components on inflammatory lung diseases-a literature review. Int J Food Sci Nutr 68(7):771-787

30. Baker JF, Sauer BC, Cannon GW et al (2016) Changes in body mass related to the initiation of disease-modifying therapies in rheumatoid arthritis. Arthritis Rheumatol 68(8):1818-1827

31. Baughman RP, Winget DB, Lower EE (2000) Methotrexate is steroid sparing in acute sarcoidosis: results of a double blind, randomized trial. Sarcoidosis Vasc Diffuse Lung Dis 17(1):60-66 
32. Singh G, Fries JF, Spitz P, Williams CA (1989) Toxic effects of azathioprine in rheumatoid arthritis. A national post-marketing perspective. Arthritis Rheum 32(7):837-843

33. Baughman RP, Drent M, Kavuru M et al (2006) Infliximab therapy in patients with chronic sarcoidosis and pulmonary involvement. Am J Respir Crit Care Med 174(7):795-802

34. European Medicines Agency INN-Infliximab (2019) https://www. ema.europa.eu/en/documents/product-information/remicade-eparproduct-information_nl.pdf. Accessed 3 Dec 2019

35. Sfriso P, Caso F, Filardo GS et al (2016) Impact of 24 months of anti-TNF therapy versus methotrexate on body weight in patients with rheumatoid arthritis: a prospective observational study. Clin Rheumatol 35(6):1615-1618

36. Lutf A, Hammoudeh M (2012) Weight gain and hair loss during anti-TNF therapy. Int $\mathrm{J}$ Rheumatol. https://doi. org/10.1155/2012/593039

37. Angelakis E, Million M, Kankoe S, Lagier JC, Armougom F, Giorgi R, Raoult D (2014) Abnormal weight gain and gut microbiota modifications are side effects of long-term doxycycline and hydroxychloroquine treatment. Antimicrob Agents Chemother 58(6):3342-3347
38. Wang T, Glendinning J, Grushka M, Hummel T, Mansfield K (2017) From the cover: drug-induced taste disorders in clinical practice and preclinical safety evaluation. Toxicol Sci 156(2):315-324

39. Schiffman SS (2018) Influence of medications on taste and smell. World J Otorhinolaryngol Head Neck Surg 4(1):84-91

40. Doty RL, Shah M, Bromley SM (2008) Drug-induced taste disorders. Drug Saf 31(3):199-215

41. Proesmans VLJ, Drent M, Elfferich MDP, Wijnen P, Jessurun NT, Bast A (2019) Self-reported gastrointestinal side effects of antifibrotic drugs in Dutch idiopathic pulmonary fibrosis patients. Lung 197(5):551-558

Publisher's Note Springer Nature remains neutral with regard to jurisdictional claims in published maps and institutional affiliations. 